Gözde Dursun*, Saurabh Balkrishna Tandale, Jörg Eschweiler, Mersedeh Tohidnezhad, Bernd Markert, and Marcus Stoffel

\title{
Recognition of tenogenic differentiation using convolutional neural network
}

https://doi.org/10.1515/cdbme-2020-3051

\begin{abstract}
Methodologies to assess stem cell differentiation in the culturing state are needed for regenerative medicine and tissue engineering techniques. In recent years, convolutional neural networks (CNNs), a class of deep neural networks, have made impressive advancements in image-based classification, recognition and detection tasks. CNNs have been introduced as a non-invasive cell characterization method by learning features directly from image data of unlabeled cells. Furthermore, this approach serves as a rapid and inexpensive methodology with high performance compared to traditional techniques that require complex laboratory procedures including antibody staining and gene expression analysis. Here, we studied the potential of the CNNs approach to recognize stem cell differentiation based on cell morphology utilizing phasecontrast microscopy images. We have examined the differentiation potential of bone marrow mesenchymal stem cells (BM$\mathrm{SCs}$ ) into tenocytes, with the treatment of bone morphogenetic protein-12 (BMP-12). After treatment, the phase-contrast images of cells were obtained directly from cell culture flasks to train $\mathrm{CNN}$ and the differentiated phenotype of stem cells was characterized by immunostaining. $\mathrm{CNN}$ was able to classify the cells into three groups including non-stem cells (chondrocytes), stem cells (BMSCs) and differentiated stem cells (tenocytes) based on their morphology with $92.2 \%$ accuracy. The presented study revealed that CNN performed faster and non-invasive cell classification task compared to traditional methodologies.
\end{abstract}

Keywords: Stem cell differentiation, cell classification, convolutional neural network

\footnotetext{
*Corresponding author: Gözde Dursun, Institute of General Mechanics, RWTH Aachen University, Aachen, Germany, dursun@iam.rwth-aachen.de

Saurabh Balkrishna Tandale, Institute of General Mechanics, RWTH Aachen University, Aachen, Germany

Jörg Eschweiler, Department of Orthopaedics, RWTH Aachen University, Aachen, Germany

Mersedeh Tohidnezhad, Institute of Anatomy and Cell Biology, RWTH Aachen University, Aachen, Germany

Bernd Markert, Institute of General Mechanics, RWTH Aachen University, Aachen, Germany

Marcus Stoffel, Institute of General Mechanics, RWTH Aachen University, Aachen, Germany
}

\section{Introduction}

Regenerative medicine and tissue engineering techniques utilize bone marrow mesenchymal stem cells (BMSCs) to promote the regeneration of damaged or diseased tissues and organs. BMSCs are undifferentiated cells with self-renewal potential, differentiation into several types of cells $[1,2]$. Cell characterization analysis after inducing stem cell differentiation have mostly performed with antibody staining, gene expression analysis, etc. These invasive methods are timeconsuming, costly, and limiting clinical translation by consuming cellular material which would be useful for cell therapy. Therefore, non-invasive methods are needed for recognition of cellular differentiation based on cell morphology in culturing state for advanced clinical outcomes [3, 4].

Deep neural networks, particularly the convolutional neural networks (CNNs), are widely applied in challenging imagebased classification by extracting image features from image pixels [5]. Many applications of deep neural networks have been reported in the field of cell biology including classification of myogenic $\mathrm{C} 2 \mathrm{C} 12$ cells at differentiation [4] and red blood cells in sickle cell anemia [6], prediction of osteogenic differentiation potential [3], classification of intracellular actin networks [7], and evaluation of human-induced pluripotent stem cell [8]. CNNs learn features from image data causing any invasion. Additionally, CNNs can serve as a rapid methodology with high performance for cell recognition [9].

The present study aimed to utilize the CNNs approach for recognition of tenogenic differentation. BMSCs differentiation was induced using different concentrations and incubation periods of BMP-12 which has been reported to induce tendon formation and has already made some achievements in the field of tendon injuries [10]. The differentiated phenotypes of BMSCs were characterized by immunostaining of tenomodulin as a tenogenic-specific marker to create the classification groups of CNN. The CNN was trained with a large data-set of phase-contrast microscopy images from three different types of cells including chondrocytes (as non-stem cells), BMSCs and tenocytes differentiated from BMSCs. The accuracy was checked using a testing data set. 


\section{Materials and methods}

\subsection{Stem cell culture and growth factor treatment}

BMSCs at passage three were cultivated for the experiments in different petri dishes with a density of approximately 3500 cells $/ \mathrm{cm}^{2}$. After 70-80\% confluency, the BMSCs were first starved for 12 hours in DMEM supplemented with 1\% FBS and then treated with $10 \mathrm{ng} / \mathrm{ml}$ or $50 \mathrm{ng} / \mathrm{ml} \mathrm{BMP-12/GDF-7}$ (Sigma-Aldrich) for 12, 24 and 48 hours. The cultivation period was kept in DMEM medium supplemented with $10 \%$ FBS after the BMP-12 treatments. Control group was cultured without BMP-12 treatment. The chondrocyte cell line (Promocell, Heidelberg, Germany) was chosen as a negative control and they were cultured in a similar manner without treatment.

\subsection{Immunofluorescence analysis}

The protein expression of biomarkers to characterize the differentiated phenotype of stem cells was analyzed by immunostaining. Briefly, the cells cultured on the cover slips were fixed with $4 \%$ formalin for 30 minutes followed by permeabilization with $0,1 \%$ Triton-X in PBS for 10 minutes. The primary antibody against tenomodulin (Tnmd) (Santa Cruz Biotechnology) was used in a dilution of 1:200. A corresponding fluorochrome-conjugated secondary antibody (Alexa-Fluor 555, Invitrogen) with a dilution of 1:250 was used to bind the primary antibody. Bisbenzimide staining was used to counter-stain the nuclei. Fluorescence images were acquired with identical exposure times using a Keyence BZ-9000 microscope with a 20X PlanFlour El NA $0.45 \mathrm{Ph} 1$ objective. The fluorescence intensity was quantified using ImageJ image processing program and the results are expressed as mean fluorescence intensity. Negative control was maintained in a similar manner to detect background fluorescence and to fix the exposure time. Statistical significance was evaluated by ttest between the untreated group and each treated group using GraphPad Prism 8.0 software.

\subsection{Acquisition of light microscopy images and data augmentation}

The cells in all the treated groups, control and negative group were washed with phosphate-buffered saline (PBS) prior to image acquisition. The light microscopy images obtained with equal light intensity using a light microscope (Leica) with a 10X magnification in size of 1280x960 pixels. Each group contains around 120 pictures. Prior to training of the images with $\mathrm{CNN}$, the data augmentation was performed in order to create more images by resizing, zooming, rotating. This ap- proach enables the model capture more features to increase accuracy and reduce overfitting $[2,7]$.

\subsection{Convolutional neural network}

Phase-contrast images of one of the treated group $(50 \mathrm{ng} / \mathrm{ml}$, 24h), control and negative groups were divided into a training and a validation set. The CNN was built based on the VGG16 architecture using Keras library and Tensorflow functions in Python. The original size of images was initially reduced to 64x64 pixels and then fed into the neural network. The network consists of 10 convolutional layers followed by 4 maxpooling layers, one flatten layer and 2 dense layers. Lastly, a softmax layer containing 3 neurons was applied to classify the cells into three different classes: BMSCs (Control,0), chondrocytes (Negative,1), and treated group which are tenocytes differentiated from BMSCs (Positive,2).

The rectified linear unit ( $\mathrm{ReLu})$ was applied as a nonlinear activation function to improve the performance of a CNNs [5], as well as it is the default activation for CNNs. The function will output the input directly if it is positive (2), otherwise, it will output zero (1).

$$
A\left(\text { net }_{j}\right)=\max (0, n e t) \begin{cases}0, & \text { if } \text { net }_{j}<0 \\ \text { net }_{j}, & \text { if } \text { net }_{j} \geq 0\end{cases}
$$

Batch normalization was adopted following each activation to enable each layer of the network to learn individually, and independently of other layers. This approach accelerates training by dramatically reducing the number of epochs and provides some regularization by reducing generalization error [11].

Categorical cross-entropy loss was implemented as a loss function to measure the performance of the model. It is a combination of softmax activation (3) and cross-entropy loss and used for multi-class classification. Softmax is frequently used in the last dense layer for multi-classification applications in CNNs.

$$
s\left(x_{i}\right)=\frac{e^{x_{i}}}{\sum_{j=1}^{n} e^{x_{j}}}
$$

where $\mathrm{x}_{i}$ indicates the output from the last dense layer and $\mathrm{n}$ denotes the total number of components in the vector. The softmax function, s, turns the numeric output into probabilities and normalizes them to form a probability distribution over classes. These probability values are used to compute the cross-entropy loss, as follows:

$$
H=-\sum_{i}^{n} p_{i} \log \left(s\left(x_{i}\right)\right)
$$

where $\mathrm{p}$ denotes the desired output. $\mathrm{H}$ is the categorical cross-entropy. 


\section{Results}

\subsection{Effects of growth factor on tenocyte differentation}

The relative fluorescence intensity was calculated by measuring integrated density using ImageJ image processing program and subtracting the mean integrated density value of negative control.
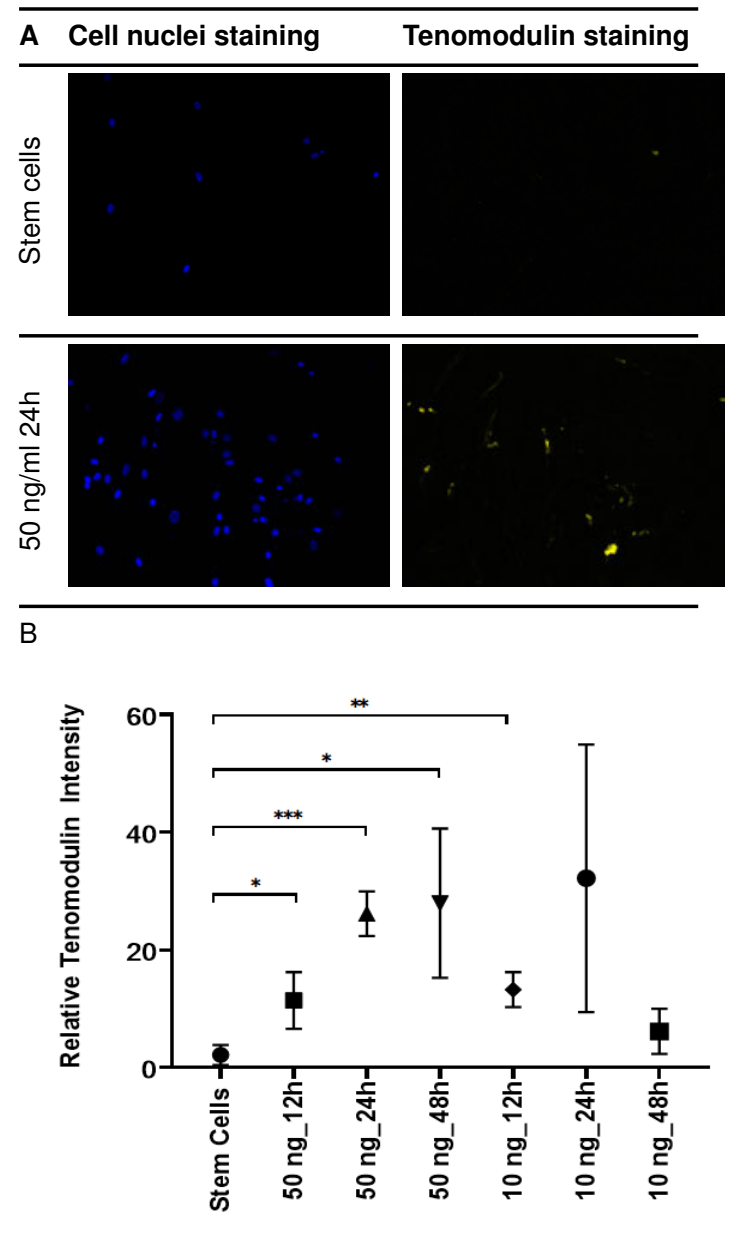

Fig. 1: A. Immunofluorescence analysis for the expression of tenomodulin in a representative sample. B. Tenomodulin expression increased most significantly in the group treated with 50 ng/ml BMP-12 for 24 h. (no star: non-significant, ${ }^{*} \mathrm{P}<0.05$, ${ }^{* *} \mathrm{P}<0.01$ and $\left.{ }^{* * *} P<0.001\right)$.

The tenomodulin expression of cells was significantly increased in the groups treated with $50 \mathrm{ng} / \mathrm{ml}$ BMP-12 for 12 $(\mathrm{p}=0.0351), 24(\mathrm{p}=0.0006)$ and 48 hours $(\mathrm{p}=0.0251)$, as well as with $10 \mathrm{ng} / \mathrm{ml} \mathrm{BMP-12}$ for 12 hours $(\mathrm{p}=0.0050)$ when compared with corresponding untreated control group. The cell colonies in these groups were differentiated into tenocytes. The groups treated with $10 \mathrm{ng} / \mathrm{ml}$ BMP-12 for $12(\mathrm{p}=0.0845)$ and 24 hours $(\mathrm{p}=0.1752)$ did not show any significant change (see Figure 1), meaning that the cell colonies in these two groups were kept their stem cell behaviours. The group treated with $50 \mathrm{ng} / \mathrm{ml}$ BMP-12 for 24 hours was chosen as the third class of $\mathrm{CNN}$, since the cells in this group showed the most significant tenomodulin expression. The five remaining experiment group was used to examine the $\mathrm{CNN}$ model for prediction.

\subsection{Performance of the convolutional neural network}

Data augmentation enabled us to generate around 3000 more images of each class. In the end, the training data set consisted of 7500 pictures and the testing data set consisted of $1500 \mathrm{im}$ ages. BMSCs (Control,0), chondrocytes (negative, 1 ) and tenocytes (positive,2) were classified using softmax function.
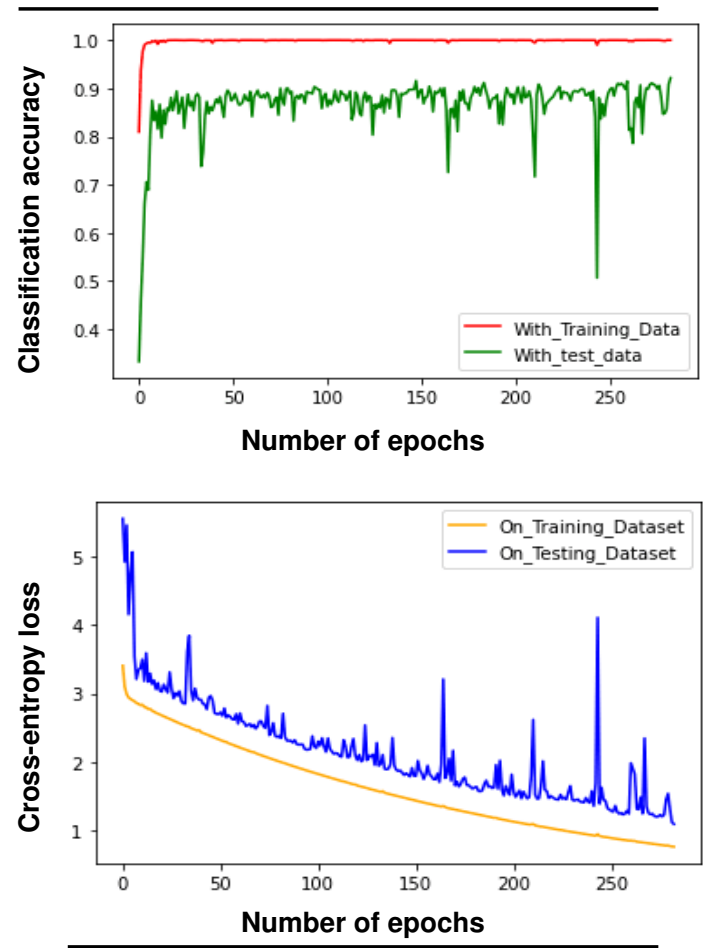

Fig. 2: Classification accuracy (up) and cross-entropy loss (down) for both training and testing across training epochs.

As presented in Figure 2, the accuracy of the trained data set achieved to $100 \%$ and the loss was minimized to 0.34 . The accuracy of the trained data set was increased from $85 \%$ to $100 \%$ using the data augmentation. The accuracy of testing data was $92.2 \%$ and loss was minimized to 1 . The early stop approach was implemented to avoid the overfitting of the model [11]. Training was stop after 273 epochs done in 1 hour 15 minutes. Although the accuracy of training and test data set was stabilized after 33 epochs, the training was kept until the 
loss values were minimized. An experiment group consisting of 44 untrained images were used to examine the model. The accuracy was $89 \%$ meaning the 39 images predicted correct (see Table1).

Tab. 1: Classification with the first experiment group. $\mathrm{n}$ shows the image number.

\begin{tabular}{lccc}
\hline $\begin{array}{l}\text { Experiment data } \\
\text { set }\end{array}$ & $\begin{array}{c}\text { BMSCs } \\
(\mathbf{n}=\mathbf{1 1})\end{array}$ & $\begin{array}{c}\text { Chondrocytes } \\
(\mathbf{n}=\mathbf{1 1})\end{array}$ & $\begin{array}{c}\text { Tenocytes } \\
(\mathbf{n}=\mathbf{2 2})\end{array}$ \\
\hline Correct prediction & 10 & 10 & 19 \\
Incorrect prediction & 1 & 1 & 3 \\
Accuracy & $91 \%$ & $91 \%$ & $86,4 \%$ \\
\hline
\end{tabular}

The phase-contrast pictures of five remaining experiment groups were used to examine the $\mathrm{CNN}$ whether it has learned the desired features for classification. Cells in the group treated with $50 \mathrm{ng} / \mathrm{ml}$ BMP-12 for 12 and 48 hour, as well as $10 \mathrm{ng} / \mathrm{ml}$ BMP-12 for 12 were classified as tenocytes with above $55 \%$ accuracy which is in good agreement with the results of immunofluorescence analysis. Cells in the group treated with 10 ng/ml BMP-12 for 48 hours and characterized as stem cells by immunofluorescence were classified also with $\mathrm{CNN}$ as stem cells with an accuracy of $70 \%$. The group treated with 10 $\mathrm{ng} / \mathrm{ml}$ BMP-12 for 24 hours was not recognized well which is in agreement with the tenomodulin expression analysis not showing any significant change with a high standard error (see Figure 1 and Table 2).

Tab. 2: Cell recognition examination of the convolutional neural network model. ${ }^{*}$ shows cell characterization based on immunofluoresence antibody staining.

\begin{tabular}{|c|c|c|c|}
\hline Groups & BMSCs & Chondrocytes & Tenocytes \\
\hline $\begin{array}{l}10 \mathrm{ng} / \mathrm{ml} 12 \mathrm{~h} \\
\text { ('Tenocytes) }\end{array}$ & $27 \%$ & $14 \%$ & $58 \%$ \\
\hline $\begin{array}{l}10 \mathrm{ng} / \mathrm{ml} \quad 24 \mathrm{~h} \\
\left({ }^{*} \mathrm{BMSCs}\right)\end{array}$ & $21 \%$ & $30 \%$ & $49 \%$ \\
\hline $\begin{array}{l}10 \mathrm{ng} / \mathrm{ml} \quad 48 \mathrm{~h} \\
\left({ }^{*} \mathrm{BMSCs}\right)\end{array}$ & $70 \%$ & $10 \%$ & $20 \%$ \\
\hline $\begin{array}{l}50 \mathrm{ng} / \mathrm{ml} 12 \mathrm{~h} \\
\left({ }^{*} \text { Tenocytes) }\right.\end{array}$ & $42 \%$ & $0 \%$ & $58 \%$ \\
\hline $\begin{array}{l}50 \mathrm{ng} / \mathrm{ml} 48 \mathrm{~h} \\
\left({ }^{*} \text { Tenocytes) }\right.\end{array}$ & $43 \%$ & $0 \%$ & $57 \%$ \\
\hline
\end{tabular}

\section{Discussion}

The utilization of CNNs offer a new approach for non-invasive and label-free cell characterization method. In the present study, a new CNN model was developed and introduced to recognize tenogenic differentiation with high accuracy by classifying stem cells (BMSCs) and tenocytes (differentiated BM$\mathrm{SCs}$ ) cells based on their morphologies. The classification pictures was completed time efficiently. Therefore, the introduced CNN model for image classification is a faster and cheaper approach compared to traditional cell characterization methods. Moreover, the usage of phase-contrast microscopy images taken directly from cell culture flasks increases the applicability of the CNN model in several laboratories.

Previous studies have reported that CNNs were applied for classification of different cells based on cellular morphology, labeled cellular proteins. Here the target was to distinguish stem cell differentiation during the culturing phase of cells which can potentially automate and improve the characterization of therapeutic stem cell populations which are currently based on conventional time-consuming, destructive assays and qualitative microscopic assessments.

We further showed that the $\mathrm{CNN}$ could be examined with unknown experiment groups to predict whether corresponding cells in each group are belong to stem cells or tenocytes. The results demonstrated a correlation with immunofluorescence analysis. CNN model classified the experiment groups with more than $57 \%$ accuracy, although there was no difference between the cell images which could be empirically obtained. This indicates that CNN model has tendency to learn the desired feature of cells. An application of the background removal approach could improve the performance of cell recognition.

As a conclusion, we introduced an accurate convolutional neural network to recognize cellular morphology changes due to the tenocyte differentiation process using images of labelfree live cells. This model can be implemented for any other cell differentiation process in regenerative medicine. Importantly this technique provides a fast tool to identify cells during cultivation enabling the adjustment of culture conditions to improve yield of therapeutic stem cells.

Acknowledgment: This research was funded by the Excellence Initiative of the German federal and state governments.

\section{Author Statement}

Conflict of interest: Authors state no conflict of interest. Informed consent: Informed consent has been obtained from all individuals included in this study. Ethical approval: The research related to human use complies with all the relevant na- 
tional regulations, institutional policies and was performed in accordance with the tenets of the Helsinki Declaration, and has been approved by the authors' institutional review board or equivalent committee.

\section{References}

[1] Gaspar D, Spanoudes K, Holladay C, Pandit A, Zeugolis D. Progress in cell-based therapies for tendon repair. Advanced Drug Delivery Reviews. 2015 Apr;84:240-256. DOI: 10.1016/j.addr.2014.11.023.

[2] Rajabzadeh N, Fathi E, Farahzadi R. Stem cell-based regenerative medicine. Stem Cell Investig. 2019;6:19. Published 2019 Jul 18. doi:10.21037/sci.2019.06.04

[3] Matsuoka F, Takeuchi I, Agata H, Kagami H, Shiono H, et al. (2013) Morphology-Based Prediction of Osteogenic Differentiation Potential of Human Mesenchymal Stem Cells. PLoS ONE 8(2): e55082. doi:10.1371/journal.pone.0055082

[4] Niioka, H., Asatani, S., Yoshimura, A. et al. Classification of $\mathrm{C} 2 \mathrm{C} 12$ cells at differentiation by convolutional neural network of deep learning using phase contrast images. Human Cell 31, 87-93 (2018). https://doi.org/10.1007/s13577-017-01919

[5] Stoffel M, Gulakala R, Bamer F, Markert B (2020)Artificial neural networks in structural dynamics: A new modular radial basis function approach vs. convolutional and feedforward topologies. Computer Methods in Applied Mechanics and Engineering 364 (2020) 112989

[6] Xu M, Papageorgiou DP, Abidi SZ, Dao M, Zhao H, Karniadakis GE (2017) A deep convolutional neural network for classification of red blood cells in sickle cell anemia. PLoS Comput Biol 13(10): e1005746

[7] Oei RW, Hou G, Liu F, Zhong J, Zhang J, An Z, et al. (2019) Convolutional neural network for cell classification using microscope images of intracellular actin networks. PLoS ONE 14(3): e0213626.https://doi.org/10.1371/journal. pone. 0213626

[8] Tokunaga, K., Saitoh, N., Goldberg, I. et al. Computational image analysis of colony and nuclear morphology to evaluate human induced pluripotent stem cells. Sci Rep 4, 6996 (2015). https://doi.org/10.1038/srep06996

[9] Dürr O., Sick B. (2016) Single-Cell Phenotype Classification Using Deep Convolutional Neural Networks. Journal of Biomolecular Screening 2016, Vol. 21(9) $998-1003$. doi: 10.1177/1087057116631284

[10] Wang, D., Ji, Z., Wang, J., Tan, Y. (2018). Bone morphogenetic protein-12 inducing tenogenic differentiation of mesenchymal stem cells enhances healing of linea alba incision. Experimental and Therapeutic Medicine, 16, 5067-5072. https://doi.org/10.3892/etm.2018.6861

[11] Goodfellow, lan and Bengio, Yoshua and Courville, Aaron. Deep Learning(2016). The MIT Press, 0262035618.

[12] Prechelt L. Early Stopping - But When?. In: Orr G.B., Müller KR. (eds) Neural Networks: Tricks of the Trade. Lecture Notes in Computer Science, vol 1524. Springer, Berlin, Heidelberg 